\title{
A Choropleth Map Classification Scheme with Control of Robustness for Uncertain Geographical Attributes
}

\author{
Wangshu $\mathrm{Mu}^{\mathrm{a},{ }^{*}}$, Daoqin Tong ${ }^{\mathrm{a}}$ \\ a School of Geographical Sciences and Urban Planning, Arizona State University,wangshum@asu.edu, Daoqin.Tong@asu.edu \\ * Corresponding author
}

Keywords: Choropleth Mapping, Robustness, Optimization, Uncertainty

\begin{abstract}
:
Choropleth mapping visualizes geographical data by grouping map units into a few classes and assigning different colors, shades or patterns to each class. Traditional choropleth mapping method assumes that the data to be displayed are certain. However, many geographical attributes contain uncertainty. For example, attributes generated from survey results may contain sampling error, and data summarized based on statistic inferences usually have a confidence interval. In recent years, some efforts have been made to study choropleth maps for uncertain geographical attributes. Existing research mainly focuses on identifying the class breaks that optimize the within-class homogeneity or between-class separability. These studies have paid little attention to the evaluation of the possibility that each areal unit might be placed in a wrong class due to its associated uncertainty. This paper addresses this issue by proposing a new choropleth mapping scheme that explicitly incorporates robustness. Given a user specified acceptable robustness level that defines the maximum tolerance of misplacement, this paper provides a maximum likelihood estimation-based method to determine the optimal class breaks. A discretization method is introduced to solve the optimization model and generate class breaks. The method is applied to map the American Community Survey 5-year estimated county-level income data with normally distributed uncertainty in the United States at various robustness levels. Results are compared with the theoretical lower bound to show the effectiveness of the discretization method. The relationship between the proposed method and the existing approaches is discussed.
\end{abstract}

